\title{
DGPS Enhancement to GPS NMEA Output Data: DGPS by Correction Projection to Position-Domain
}

\author{
Byungwoon Park ${ }^{1}$, Jeongkeun $\mathrm{Lee}^{2}$, Younsil Kim ${ }^{3}$, Ho Yun ${ }^{3}$ \\ and Changdon $\mathrm{Kee}^{3}$ \\ ${ }^{1}$ (School of Mechanical and Aerospace Engineering, Sejong University, Seoul, Korea) \\ ${ }^{2}$ (Networking \& Communications Lab, Hewlett-Packard Labs, Palo-Alto, USA) \\ ${ }^{3}$ (GNSS Lab. School of Mechanical and Aerospace Engineering and SNU-IAMD, \\ Seoul National University, Korea) \\ (E-mail: kee@snu.ac.kr)
}

\begin{abstract}
Most Differential Global Positioning System (DGPS) correction formats are based on range information, and thus typical DGPS systems can be implemented only on correction message-readable or raw observable-providing devices. There is no other way to improve an already-calculated position than a 'block shift technique', which has a very limited applicability. This paper suggests an algorithm to project measurement correction directly to position domain data without requiring raw pseudorange data. By post-processing methodology, we evaluated the performance of our new algorithm compared to conventional DGPS, which requires raw pseudorange data; the observed difference between them was only $0.1 \mathrm{~mm}$. The proposed correction projection algorithm can be used with commercial off-theshelf receivers that provide National Marine Electronics Association (NMEA) format data. Our testing with a U-blox LEA-5H receiver resulted in a drastic reduction of horizontal Root Mean Square (RMS) error from $4.75 \mathrm{~m}$ to $1.09 \mathrm{~m}$.
\end{abstract}

\section{KEY WORDS}

$\begin{array}{ll}\text { 1. Differential Global Positioning System (DGPS). } & \text { 2. National Marine Electronics }\end{array}$ Association (NMEA). 3. Correction Projection. 4. Position-domain DGPS.

Submitted: 14 February 2012. Accepted: 6 September 2012. First published online: 23 October 2012.

1. INTRODUCTION. Differential Global Positioning System (DGPS) is an enhancement to Global Positioning System (GPS); DGPS uses fixed ground-based reference station coordinates to correct user's position accurately by broadcasting the differential correction. Two different methodologies are used to implement a real-time DGPS service, namely: 'block shift technique' and 'range correction'. Corrections to the coordinates of the 'block shift technique' are made by comparing a known reference station position and instantaneously computing the corrected position. 'Range correction' technique generates corrections to all pseudorange by comparing 
the true to the observed range, based on the reference station coordinates. The block shift technique is the easiest method to implement, but it is available only if both the remote and base receivers use the same satellite constellation to generate their point solutions. On the other hand, the 'range correction technique' can use any combination of corrected ranges, not just the reference station constellation, so it is the most effective real-time strategy to implement DGPS systems.

Many kinds of GPS receivers and chip-sets are currently being developed and have already been released. If one has a skilled technique and a high-priced GPS receiver which can provide raw observables in real-time or read correction messages such as Radio Technical Commission for Maritime Services (RTCM), Radio Technical Commission for Aeronautics (RTCA) or Satellite-Based Augmentation System (SBAS), the user can construct a customized DGPS whose accuracy is $1-3 \mathrm{~m}$. Manufacturers are still producing end devices which operate only in a stand-alone positioning mode, which do not exploit any of those correction data or DGPS capabilities, and many chip-sets installed in car navigation kits or mobile phones cannot provide as accurate positions as DGPS results. Also geo-tagging, which is often built into digital cameras or hiking devices, does not usually provide DGPS accuracy. Even though the market share of the DGPS-disabled GPS modules is still dominant, there is no adequate way to achieve the (approximately 1 metre) accuracy of DGPS, because conventional DGPS corrections are based on the 'range correction' technique.

Considering the geometry of satellite constellation and range corrections, we can construct an equation to project all the satellites' corrections to the rover's position domain. Based on the projection algorithm, this paper suggests a new 'block shift' solution, which can correct the already-calculated stand-alone GPS result in position domain without the requirement of the same satellite constellation between the rover and the base station or the requirement of raw pseudorange data access.

2. DGPS ALGOR ITHM. GPS errors are mostly caused by satellite clock bias, and variations of ephemeris and atmospheric propagation; thus GPS errors are correlated spatially up to hundreds of kilometres range and temporally in several seconds. The principle of the DGPS algorithm is based on the spatial and temporal correlation of GPS error, and it tries to mitigate the errors by differentiating measurement or position between receivers. By placing a GPS receiver at a known position, we can estimate the combined effect of the errors. When the estimated error is delivered to a rover, the error in the rover side is cancelled out and the quality of the position result improves.

In real-time operations, DGPS uses a Reference Station (RS) at a known location to calculate and broadcast corrections to local users via radio transmission or mobile communication network (Misra and Enge, 2001). This correction can be applied in two ways - 'position-domain correction' and 'range-domain correction'.

2.1. Position-Domain DGPS Correction. Conceptually, the simplest way to implement DGPS is to place the GPS reference receiver at a surveyed location, to compute the coordinate differences (in latitude, longitude, and geodetic height) between the GPS-derived position and the surveyed location, and then to transmit these differences to the user, as shown in Figure 1. To calculate the coordinate 


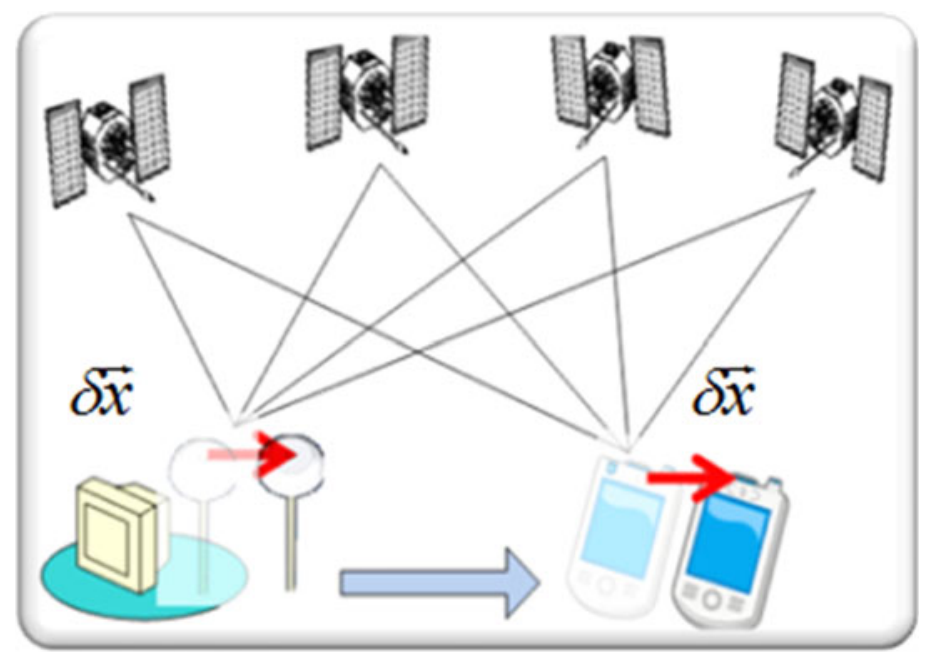

Figure 1. Position-domain DGPS.

difference $(\delta \vec{x})$, the RS continuously computes its own position according to Equation (1).

$$
\hat{\vec{x}}_{R S}=\left(H^{T} H\right)^{-1} H^{T} \vec{\rho}_{R S}
$$

where,

$\hat{\vec{x}}_{R S}$ : epoch-by-epoch estimated position of the RS

$H$ : $\quad$ GPS observation matrix

$\vec{\rho}_{R S}: \quad$ GPS pseudorange measurement set at the RS

The RS compares the instantly calculated position with its own fixed known position in the World Geodetic System 1984 (WGS84) format or in local datum coordinates, and generates a correction by differentiating them as expressed in Equation (2).

$$
\delta \vec{x}=\vec{x}_{R S, \text { fixed }}-\hat{\vec{x}}_{R S}
$$

where,

$\vec{x}_{R S, \text { fixed }}$ : fixed coordinates of the RS.

$\hat{\vec{x}}_{R S}$ : epoch-by-epoch estimated position of the RS.

After receiving the correction immediately from the RS, the rover mitigates the error in its stand-alone solution by Equation (3), and obtains an improved position, the position-domain DGPS result. In cases of post-processing, the rover's logged positions can be also corrected by the same method by utilizing correction data computed and stored by the RS:

$$
\vec{x}_{\mathrm{DGPS}(\mathrm{P})}=\hat{\vec{x}}+\delta \vec{x}
$$

where,

$\hat{\vec{x}}$ : epoch-by-epoch estimated stand-alone position of a rover

$\vec{x}_{\mathrm{DGPS}(\mathrm{P})}$ : position-domain DGPS result 


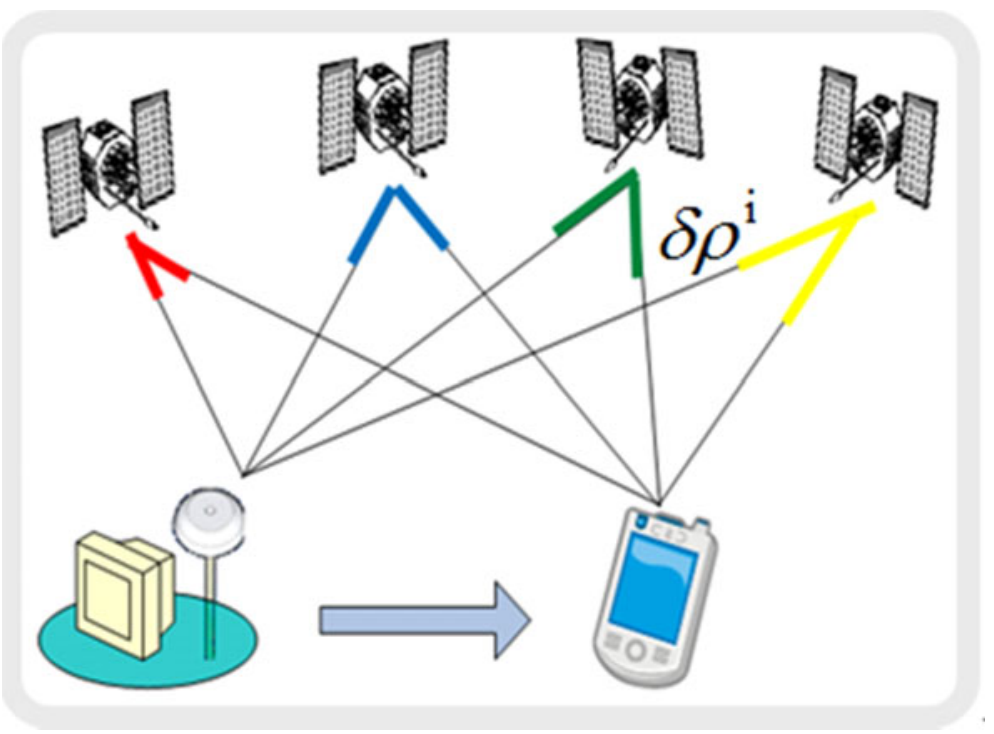

Figure 2. Measurement-domain DGPS concept.

As simple as it may sound, its usefulness in practice is small. For the most part, the coordinate differences represent the common error in the RS and the rover at the measurement time. This technique, however, requires that both the RS and the rover make pseudorange observables to the same set of satellites to ensure that common errors are experienced. Therefore, the rover must coordinate their choice of satellites with the RS, or the RS must determine and transmit position correction for all the combinations of visible satellites (Kaplan, 1996). Otherwise, severe errors can occur, possibly worse than those of the uncorrected stand-alone positioning results (Rizos, 1999).

2.2. Measurement-Domain DGPS Correction. A more effective DGPS method, 'measurement-domain DGPS', uses range corrections rather than making a shift to the coordinates. A general construction of the system is described in Figure 2.

The RS, positioned at a known location, estimates the distance to the visible satellites, and then generates the correction $\left(\delta \rho^{i}\right)$ for each satellite by differentiating using Equation (4):

$$
\delta \rho^{i}=\hat{d}_{R S}^{i}+\hat{B}_{R S}-\hat{b}^{i}-\rho_{R S}^{i}
$$

where:

$\delta \rho^{i} \quad$ pseudorange correction for i-th satellite.

$\hat{d}_{R S}^{i}: \quad$ estimated distance from $\mathrm{RS}$ to i-th satellite.

$\hat{B}_{R S}$ : estimated clock bias of RS.

$\hat{b}^{i}$ : $\quad$ estimated clock bias of $\mathrm{i}$-th satellite.

$\rho_{R S}^{i}: \quad$ RS pseudorange observable for the i-th satellite.

The correction data set $(\delta \vec{\rho})$ transmitted to the rover receiver is applied to the observables $(\vec{\rho})$, and an accurate result is calculated by least-square method in 
Equation (5):

$$
\left[\begin{array}{c}
\vec{x}_{D G P S(M)} \\
B
\end{array}\right]=\left(H^{T} H\right)^{-1} H^{T}\left[\begin{array}{c}
\vdots \\
e^{i} \cdot \vec{R}^{i}-\left(\rho^{i}+\delta \rho^{i}\right) \\
\vdots
\end{array}\right]
$$

where:

$\vec{x}_{\mathrm{DGPS}(\mathrm{M})}$ : measurement-domain DGPS result.

$H: \quad$ observation matrix.

$B: \quad$ receiver clock bias.

$e^{i} \quad$ unit vector of the line of sight direction from receiver to the i-th satellite.

$\vec{R}^{i}$ : $\quad$ vector from the receiver to the i-th satellite.

This method is much more flexible, because the rover can use any combination of the corrected ranges to obtain a solution, and not just the satellite set used at the base station. This advantage over the 'position-domain' DGPS has made the 'measurement-domain' correction popular among chipset makers and end devices, and general correction messages such as RTCM, RTCA, and SBAS (Parkinson, 1996) are based on this methodology.

3. CORRECTION PROJECTION ALGORITHM. This paper suggests a new 'block shift' solution, which can correct the already-calculated standalone GPS positions. Applying the distribute law to Equation (5), Equation (6) is obtained:

$$
\left[\begin{array}{c}
\vec{x}_{D G P S(M)} \\
B
\end{array}\right]=\left(H^{T} H\right)^{-1} H^{T}\left[\begin{array}{c}
\vdots \\
e^{i} \cdot \vec{R}^{i}-\rho^{i} \\
\vdots
\end{array}\right]+\left(H^{T} H\right)^{-1} H^{T}\left[\begin{array}{c}
\vdots \\
-\delta \rho^{i} \\
\vdots
\end{array}\right]
$$

Compared to Equation (3), the former part in Equation (6) is the estimated standalone position $(\hat{\vec{x}})$, and the latter is the correction in position domain $(\delta \vec{x})$. The relationship between the position and measurement correction can be summarized into Equation (7), where $-\left(H^{T} H\right)^{-1} H^{T}$ is the projection matrix.

$$
\delta \vec{x}=-\left(H^{T} H\right)^{-1} H^{T} \delta \vec{\rho}
$$

Equation (7) explains how the measurement correction set is converted to the position correction. Each satellite's geometry and error size make different contributions to the position, and it can be calculated by the product of the row vector of the projection matrix and the error amount for each satellite. The sum of all the satellite errors' projected value is equal to the error in the stand-alone GPS solution. After generating the position-domain correction $(\delta \vec{x})$ by Equation (7) of the newly proposed 'correction-projection' algorithm, a DGPS Correction Projection (DGPS-CP) position is obtained by applying $\delta \vec{x}$ to Equation (3).

From Equation (7) we can guess the reason why the 'block-shift correction' is valid only when the set of visible satellites at the RS and the rover are exactly the same. $\mathrm{H}$ matrix and $\overrightarrow{\delta \rho}$ consist of the terms dependent to the set of visible satellites, therefore 
Table 1. GPS Error Amount of Visible Satellite at RS for Position Error Comparison.

\begin{tabular}{lcccccccr}
\hline PRN & 11 & 7 & 8 & 17 & 19 & 20 & 28 & 32 \\
\hline Error(m) & $5 \cdot 03$ & $7 \cdot 84$ & $6 \cdot 14$ & $8 \cdot 81$ & $6 \cdot 00$ & $9 \cdot 00$ & $6 \cdot 27$ & $7 \cdot 36$ \\
Azimuth $\left(^{\circ}\right)$ & $37 \cdot 3$ & 334 & $290 \cdot 7$ & $281 \cdot 5$ & $68 \cdot 2$ & $24 \cdot 7$ & $314 \cdot 2$ & $56 \cdot 8$ \\
Elevation $\left(^{\circ}\right)$ & $81 \cdot 6$ & $23 \cdot 6$ & $40 \cdot 0$ & $20 \cdot 9$ & $33 \cdot 3$ & $24 \cdot 9$ & $46 \cdot 2$ & $30 \cdot 5$ \\
\hline
\end{tabular}

rover's different constellation from the RS makes different matrix of $\mathrm{H}$ and $\overrightarrow{\delta \rho}$, which consequently draw different position corrections.

Suppose that a RS can receive observables from eight satellites whose measurements contain errors explained in Table 1 . In this case, the position correction is $(-2.57 \mathrm{~m}$, $2.96 \mathrm{~m}, 3.65 \mathrm{~m}$ ) in the Earth-Centred Earth-Fixed (ECEF) coordinate system. If the rover receives all the measurements except of Pseudo-Random Noise (PRN) 19, the correction becomes $(-3.03,4 \cdot 77,3 \cdot 74)$ and the value of correction difference between the rover and the RS becomes $1.87 \mathrm{~m}$, which means an increased error. If the rover misses the PRN 32 signal additionally, the difference increases to $3.70 \mathrm{~m}$.

According to Payne and Carnegie (2003), standard deviations of $2.38 \mathrm{~m}$ and $4.64 \mathrm{~m}$ were recorded in latitude and longitude directions respectively without corrections, while the block-shift results were worse, $3.80 \mathrm{~m}$ and $6.90 \mathrm{~m}$. This is a significant limitation, because it is rare that the same satellite constellation is simultaneously visible if the rover is operating in a city, where the effect of urban canyons causes a serious interference of the satellite signals.

4. STRATEGY FOR DGPS-CP SYSTEM IMPLEMENTATION. In this paper, we focus on a way to improve the performance of the DGPS-disabled modules/receivers by DGPS-CP. Those DGPS-disabled devices provide Position, Velocity, Timing (PVT) results instead of GPS raw observables, and the results are generally described in the National Marine Electronics Association (NMEA) 0183 standard format. Ephemeris data is not offered in these systems, so the observation matrix $(\mathrm{H})$, which is used for the projection matrix, is not to be calculated. Considering these limitations, in this section, we describe a method to implement DGPS-CP in the off-the-shelf DGPS-disabled stand-alone GPS modules.

4.1. NMEA 0183 Standard Format. NMEA has developed the specification that defines the interface between various pieces of marine electronic equipment (NMEA, 2011). The standard permits marine electronics to send information to computers and to other marine equipment. Communication of GNSS receivers and chipsets is defined within this specification, and most computer programs understand and expect data in the NMEA format.

Each line of NMEA data begins with a ' $\$$ ' and ends with a carriage return. The data contained within a single line is expressed in ASCII and separated by commas. The first word, called a data type, defines the interpretation of the rest of the sentence. For example, GPGGA indicates GPS position fix data, while GPGLL is latitude and longitude data. Table 2 summarizes major NMEA data types.

The most important NMEA data type is the GPGGA that provides current position fix data. It includes current time, position, fix quality, and other information on the navigation. Where a numeric latitude or longitude is given, the two digits immediately 
Table 2. NMEA 0183 data description (Mehaffey et al., 2011).

\begin{tabular}{|c|c|c|}
\hline Data Type & Description & Example \\
\hline GPGGA & GPS fix data & $\begin{array}{l}\text { \$GPGGA, } 123519,4807 \cdot 038, \mathrm{~N}, 01131 \cdot 000, \mathrm{E}, 1,08,0 \cdot 9,545 \cdot 4 \text {, } \\
\text { M,46•9,M, } * 47\end{array}$ \\
\hline GPGLL & $\begin{array}{l}\text { Geographic position, } \\
\text { latitude/longitude }\end{array}$ & \$GPGLL,4916·45,N,12311·12,W,225444,A, *1D \\
\hline GPGSV & GPS Satellites in view & $\begin{array}{l}\text { \$GPGSV, } 2,1,08,01,40,083,46,02,17,308,41,12,07,344,39,14, \\
22,228,45^{*} 75\end{array}$ \\
\hline GPGSA & $\begin{array}{l}\text { GPS DOP and active } \\
\text { satellites }\end{array}$ & \$GPGSA,A,3,04,05,,09,12,,,24,,,,,2·5,1·3,2·1*39 \\
\hline
\end{tabular}

to the left of the decimal point are whole minutes, to the right are decimals of minutes, and the remaining digits to the left of the whole minutes are whole degrees. For example, if a receiver make a sentence as '\$GPGGA, 123519, 4807.038, N, 01131.000, E, 1, 08, 0, 9, 545.4,M,49.9,M, ${ }^{*} 47^{\prime}$, it means that the latitude and longitude of the GPS position fixed at 12:51:19(UTC) is $48^{\circ} 07 \cdot 038^{\prime}$ North and $11^{\circ} 31 \cdot 000^{\prime}$ East.

There are two kinds of sentences that describe current satellite status, which are GPGSV and GPGSA. Among them, GPGSA sentence provides the PRN numbers of the satellites being used in the current solution and the Dilution of Position (DOP), while GPGSV shows data about the satellites that the rover might be able to find based on its satellite viewing mask and almanac settings.

The $\mathrm{H}$ matrix consists of Line Of Sight (LOS) vectors whose initial point is the device's position. To construct the $\mathrm{H}$ matrix, we need to know which satellites are used to calculate the current position. Therefore, to compute the $\mathrm{H}$ matrix, current position and visible satellite information are needed and they are available in the GPGGA and GPGSA data in the NMEA format.

4.2. Projection Matrix Construction. LOS in the $\mathrm{H}$ matrix is the direction cosine of the vector drawn from the estimated receiver location to each satellite. The receiver's location is obtained from the GPGGA data, and the satellite PRN is easily distinguished from the GPGSA data. The only remaining job is estimating each satellite's position, although typical stand-alone GPS receivers do not provide ephemeris data. Using azimuth and elevation information of GPGSV or receiving each satellite position calculated from a $3^{\text {rd }}$ party server every epoch is possible, and getting ephemeris data via external channel in the same way as Assisted GPS (A-GPS) is a practical method. While GPS receivers generally calculate satellite positions iteratively using ephemeris, received time and raw observable data, in this paper we use only ephemeris and time data, therefore we need to consider the difference of the calculated satellite position and its effect on the DGPS position. Figure 3 shows the DGPS position difference between these two satellite position calculation methods. The difference is only $10^{-3} \mathrm{~mm}$, which is negligible in a code-based positioning.

4.3. DGPS-CP System Design. Based on the DGPS-CP basic algorithm and the implementation strategy, we construct a system as shown in Figure 4. External data channels such as Digital Multimedia Broadcasting (DMB), Code Division Multiple Access (CDMA), Global System for Mobile communication (GSM), and Wireless Local Area Network (WLAN) can deliver both ephemeris and range correction data in real-time. In the post-processing case, this data can be easily added to the logged NMEA data. After receiving ephemeris data, we can calculate the approximate 


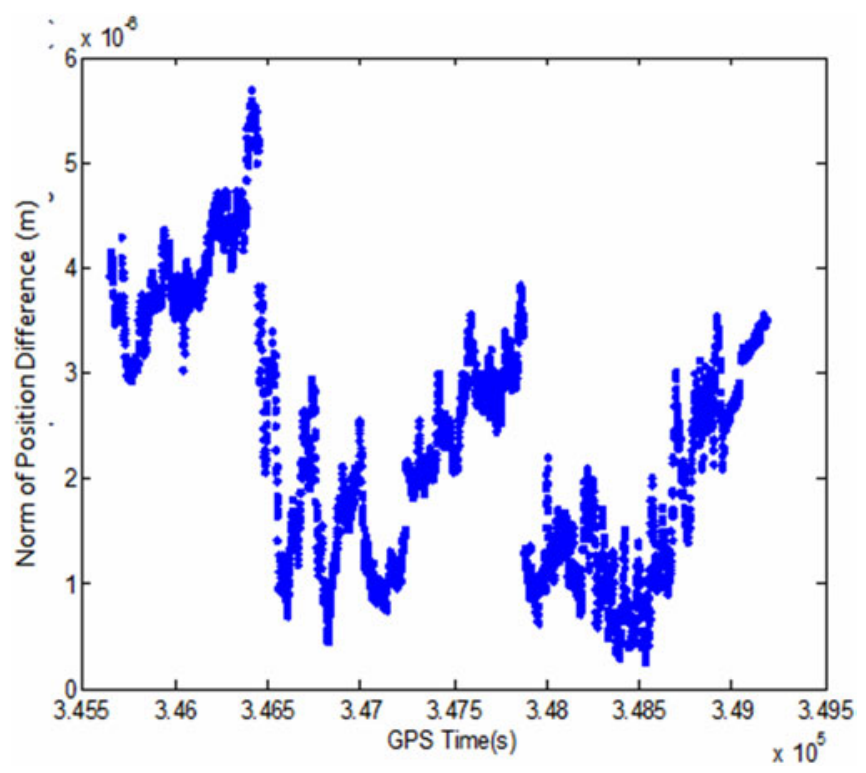

Figure 3. Norm of position difference between approximate and iteratively computed $\mathrm{H}$ matrix.

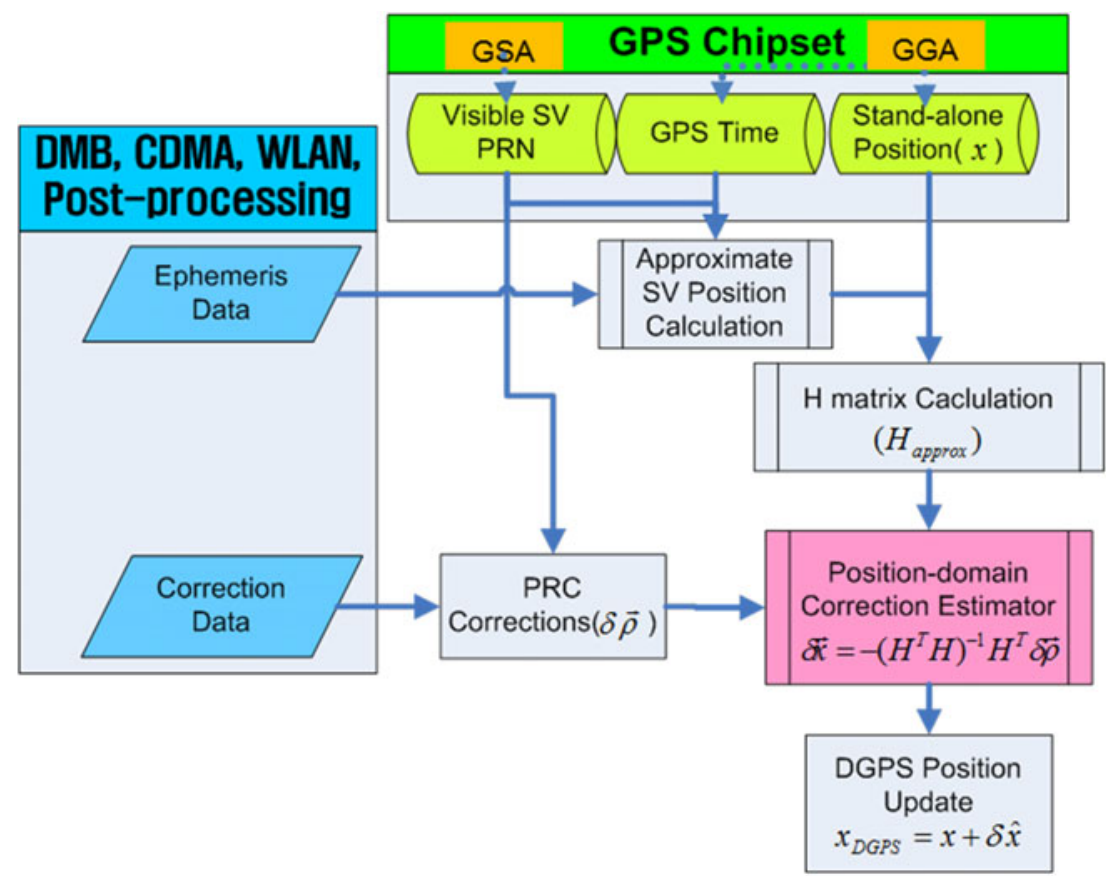

Figure 4. DGPS-CP system construction.

positions of satellites which are identified by GPGSA information at the GPS Time shown in the GPGGA message. The $\mathrm{H}$ matrix is constructed by differentiating the approximate satellite positions and stand-alone GPS result in GPGGA sentences, and 


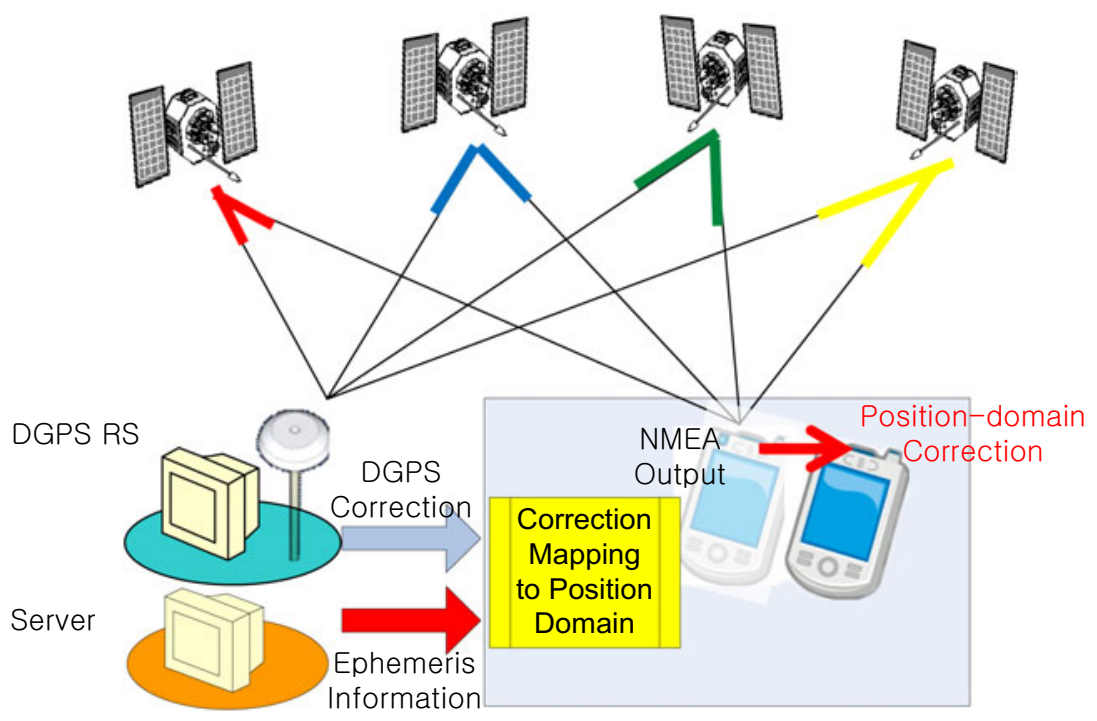

Figure 5. An example of correction mapping DGPS.

Pseudo Range Correction (PRC) set for the current satellite constellation is made by selecting correction based on the GPGSA message. Now that the H matrix and PRC Correction are set, we can project the range correction to the position domain by Equation (7) and then get the DGPS solution by Equation (3).

As described in the previous paragraph, external data inputs of DGPS range correction and ephemeris data need to be provided for a real time DGPS-CP service. The correction message for DGPS-CP is exactly same as that of the current DGPS, so the current data streams such as Networked Transport of RTCM via Internet Protocol (NTRIP), National DGPS (NDGPS) message from beacon, and SBAS messages are available without adding new servers. For a server transmitting ephemeris data, we consider A-GPS servers of cellular phone or File Transfer Protocol (FTP) services of broadcast ephemeris files as described in Figure 5.

\section{DGPS-CPALGORITHM VALIDATION USING GPS RAW DATA.}

5.1. Post-processing Test Construction. To validate the DGPS-CP algorithm, we used GPS raw data from two GPS Continuously Operating Reference Station (CORS) sites at Seoul, Korea, which were logged for Receiver Independent Exchange Format (RINEX) from 12:00 to 19:00 (Korean Standard Time [KST]) on January $3^{\text {rd }} 2011$ with $15 \mathrm{~s}$ intervals. In this post-processing test, we assumed the YONS (Yongsan) CORS site as a DGPS rover and the GUMC (Geumcheon) site as a RS. Distance between the two sites is $12.5 \mathrm{~km}$ as shown in Figure 6; we set the mask angles of the RS and the rover to $0^{\circ}$ and $15^{\circ}$ respectively, considering that the constellations at the RS and the rover can be different. The receivers and antennas of both sites were Trimble NetR5 and Zephyr Geodetic II respectively, which provide unfiltered and unsmoothed pseudorange data.

We processed the measurement of GUMC to generate PRC in RTCM standard, and then corrected the GPS frequency L1 observables of YONS. We used the same 


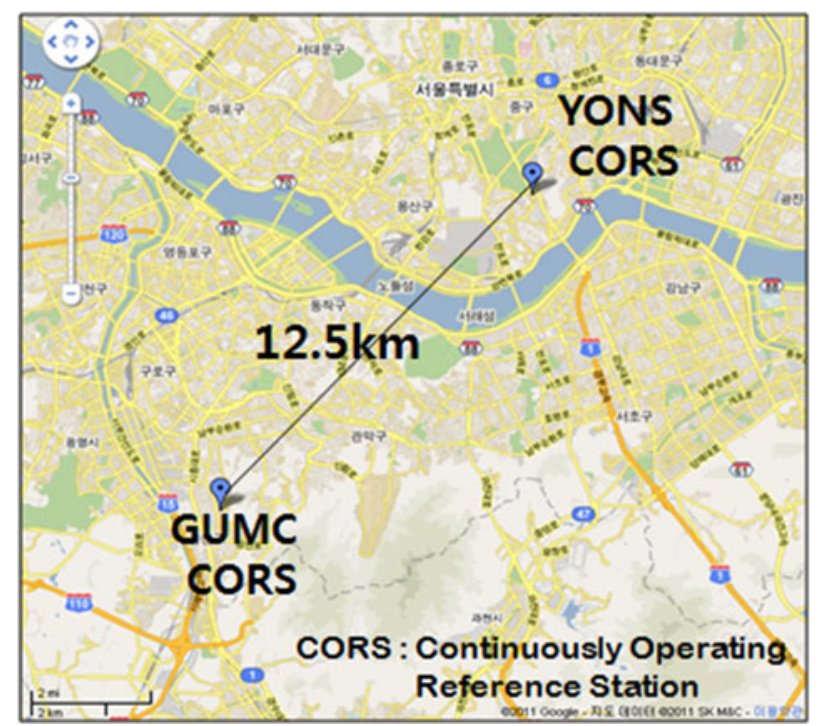

Figure 6. CORS sites used for the DGPS-CP algorithm validation.

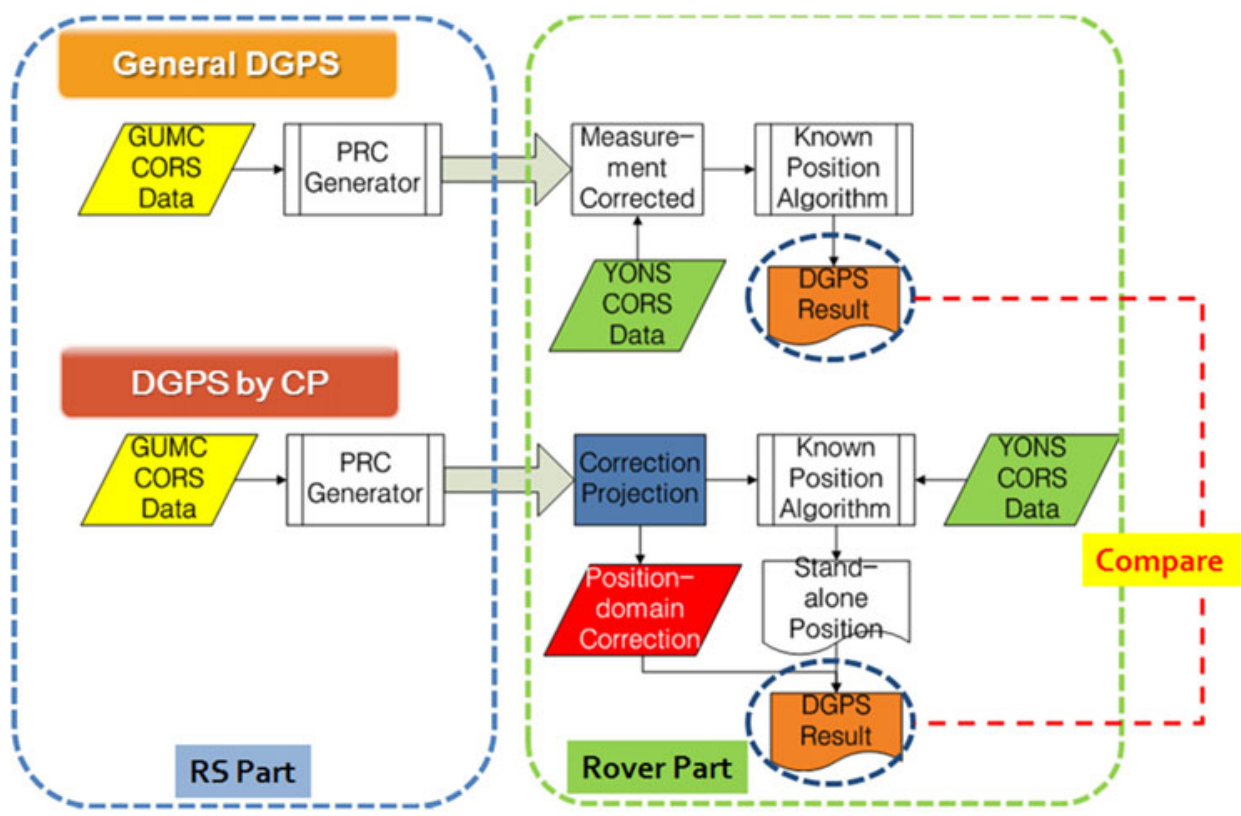

Figure 7. DGPS performance comparison process.

data in both the general DGPS and the DGPS-CP methods, and then compared the results as shown in Figure 7. To minimize the effect of other environmental factors, the same stand-alone GPS positioning algorithm was used in both methods. Note that the positioning process is performed before the error correction in the DGPS-CP case, while the general DGPS operates in a reverse order. 
Table 3. Statistics of stand-alone position at YONS CORS site.

\begin{tabular}{|c|c|c|c|c|}
\hline & \multicolumn{2}{|c|}{ Horizontal Error } & \multicolumn{2}{|c|}{ Vertical Error } \\
\hline & mean $(\mathrm{m})$ & $\mathrm{RMS}(\mathrm{m})$ & mean $(\mathrm{m})$ & $\mathrm{RMS}(\mathrm{m})$ \\
\hline Stand-alone & $0 \cdot 97$ & $1 \cdot 72$ & $7 \cdot 98$ & $8 \cdot 47$ \\
\hline
\end{tabular}
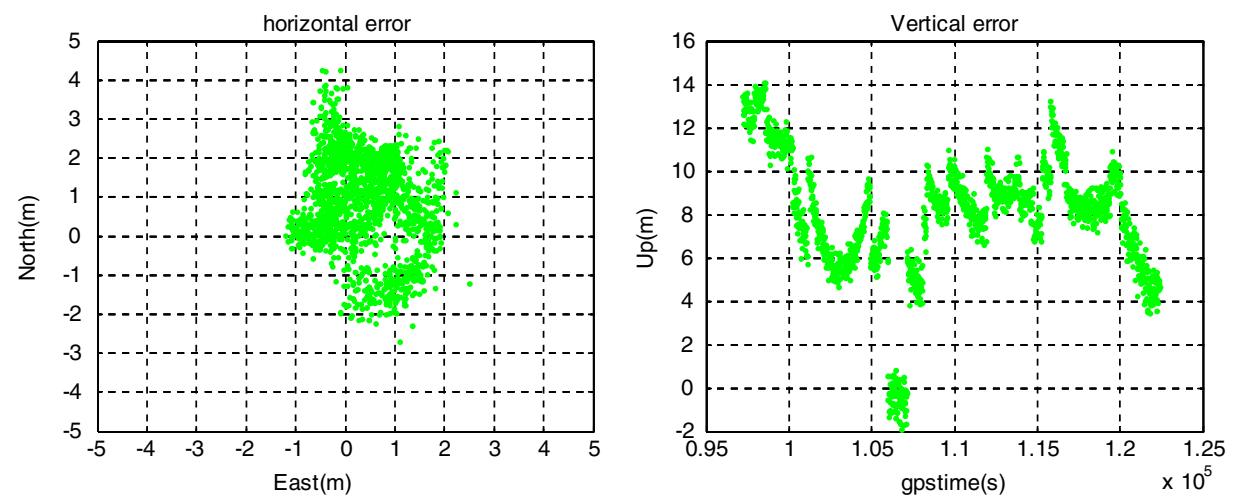

Figure 8. Stand-alone positioning results at YONS CORS site (left: horizontal, right: vertical).
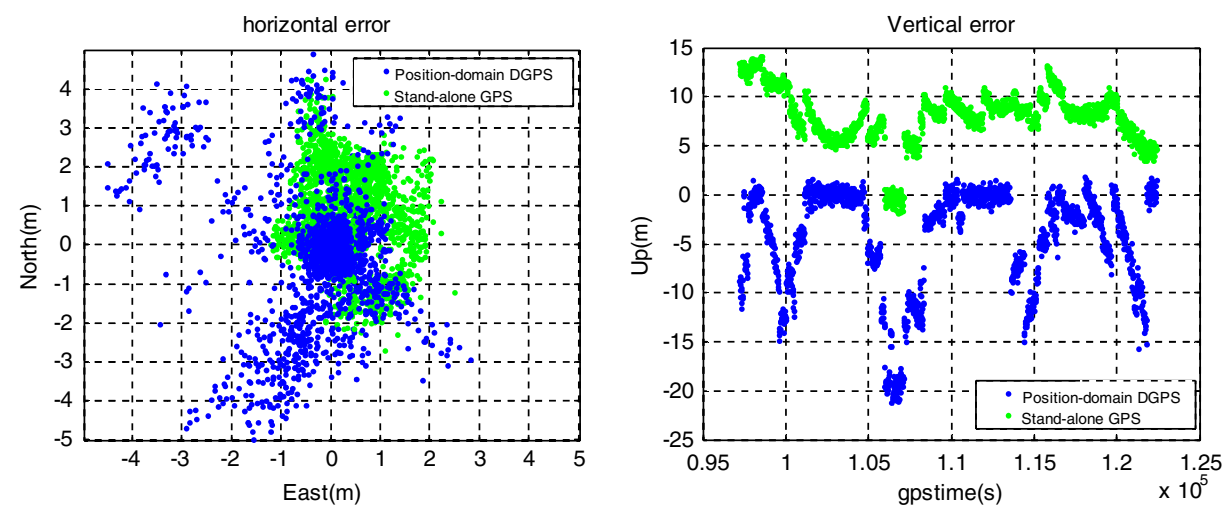

Figure 9. Block-shift DGPS results at YONS CORS (left: horizontal, right: vertical).

5.2. Stand-alone and Block-shift DGPS Results. Figure 8 and Table 3 show the stand-alone positioning result of YONS. While mean value of horizontal error is less than $1 \mathrm{~m}$ and RMS of it is $1.7 \mathrm{~m}$, vertical error reaches $14 \mathrm{~m}$.

We applied the traditional position-domain DGPS, block shifting the YONS standalone positioning result, and Figure 9 shows the result of this test. The horizontal error, which was bounded by $4 \mathrm{~m}$, has increased to $6 \mathrm{~m}$. And the vertical result corrected by the block-shift correction is occasionally worse than that of stand-alone and it goes up to $22 \mathrm{~m}$.

Because the rover is located $12 \cdot 5 \mathrm{~km}$ away from the RS, the satellite constellations of the two sites are not the same. The visible satellite set of the rover is usually a subset 


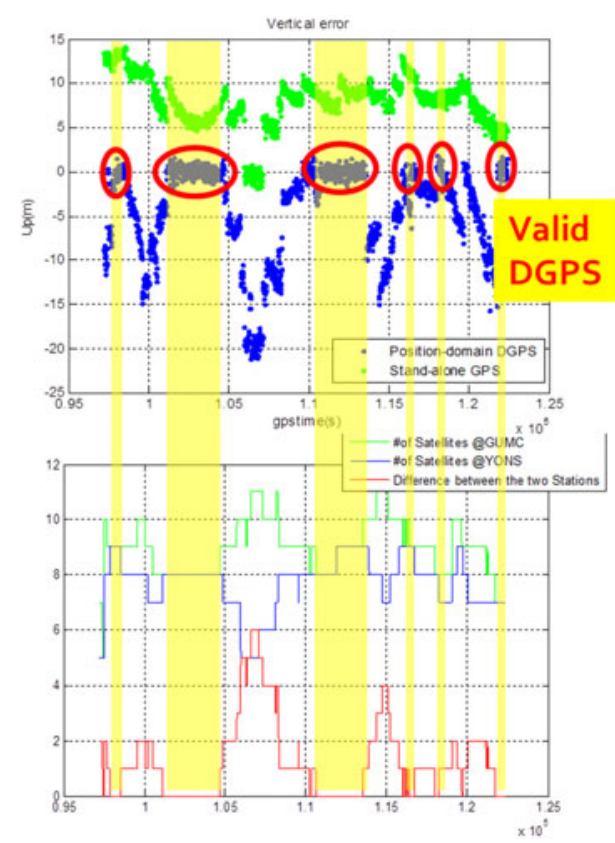

Figure 10. Correlation between block-shift DGPS performance (upper) and satellite constellation (below).
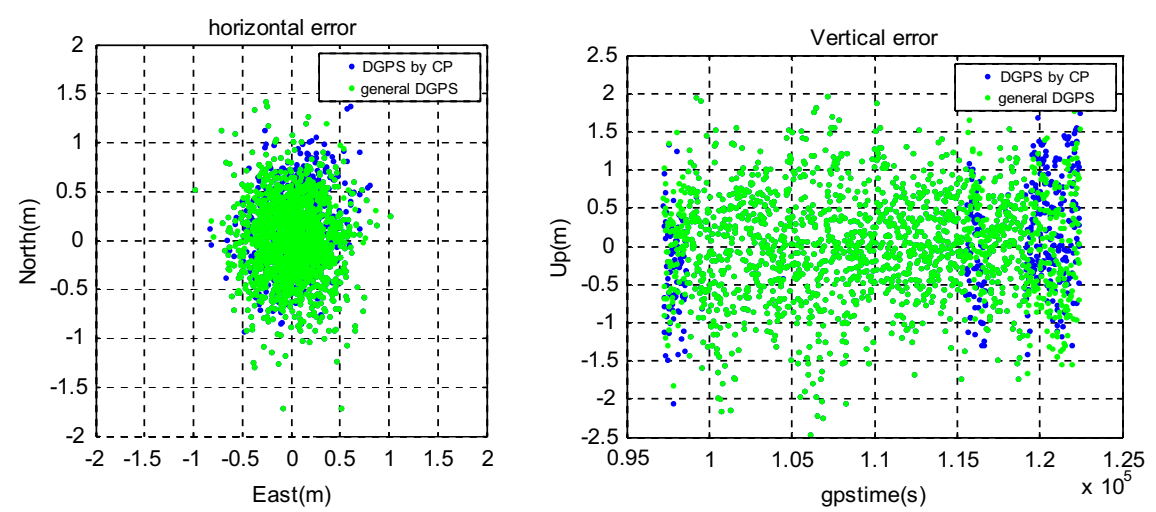

Figure 11. DGPS-CP results at YONS CORS (left: horizontal, right: vertical).

of the RS since the mask angle of the RS, $0^{\circ}$, is lower than that of the rover. In Figure 10, we have found that the DGPS is valid only when the satellite constellation of the rover is exactly same as that of the RS. Even though differentiated by the blockshift correction of the RS, the rover's position error is not fully mitigated and even enlarged when the number of visible satellite at the rover side is less than that at the RS. From the result, we can see that the block-shift method is valid only when the constellations of the RS and the rover are the exactly same.

On the other hand, the DGPS-CP yields valid results throughout the entire data process as shown in Figure 11. Because the rover can distinguish the range 
Table 4. Statistics of DGPS-CP at YONS CORS.

\begin{tabular}{lccccc}
\hline & \multicolumn{2}{c}{ Horizontal Error } & & \multicolumn{2}{c}{ Vertical Error } \\
\cline { 2 - 3 } \cline { 6 - 6 } & mean $(\mathrm{m})$ & RMS $(\mathrm{m})$ & & mean $(\mathrm{m})$ & RMS(m) \\
\hline General DGPS & 0.02 & 0.47 & & 0.02 & 0.70 \\
DGPS by CP & 0.06 & 0.50 & & 0.03 & 0.70 \\
\hline
\end{tabular}

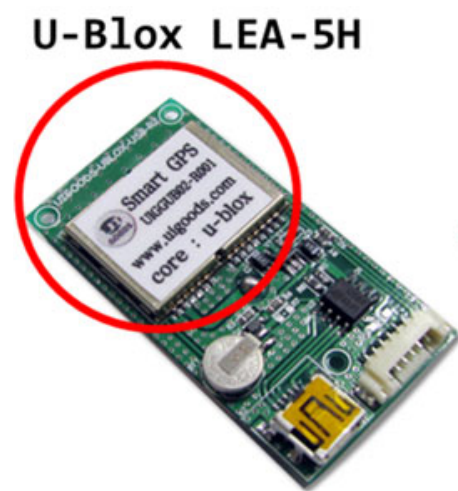

$<$ Bottom View>
Patch Antenna

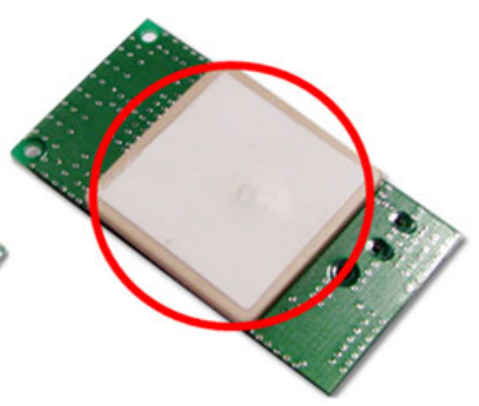

$<$ Top View $>$

Figure 12. Rover receiver module (U-blox LEA-5H).

correction of its own visible satellites among the received RTCM message from the RS and then generate the position-domain correction vector by projection, the DGPS$\mathrm{CP}$ results are almost same as that of the traditional range domain DGPS and the RMS of the differences between these two systems is only $0 \cdot 1 \mathrm{~mm}$ as summarized in Table 4.

6. FIELD TEST. To evaluate the DGPS-CP performance with a commercial off-the-shelf receiver, we conducted an epoch-by-epoch field test. For the test, we used two LEA-5H U-blox receivers with patch antennas shown in Figure 12. According to the datasheet, the accuracy of the receiver is $2.5 \mathrm{~m}$ Circular Error Probability (CEP) without correction and $2.0 \mathrm{~m}(\mathrm{CEP})$ in the SBAS DGPS mode. The receiver provides the output data of GPGGA, GPGLL, GPGSV, GPGSA in NMEA format at maximum $4 \mathrm{~Hz}$ reporting frequency, but cannot read the RTCM correction messages (UBlox Inc, 2011).

We generated the range correction from the raw data from the GUMC CORS site in the same way as the previous section, and located the receivers in the Seoul National University (SNU) campus, which is apart from the RS by $4 \cdot 1 \mathrm{~km}$ as shown in Figure 13. For fair comparison, we placed two receivers, one for the traditional DGPS and the other for DGPS-CP, under the same GPS signal re-radiator as shown in Figure 14 so that the receivers simultaneously receive the same GPS signal. We set one receiver to an SBAS-off mode to apply DGPS-CP, while the other in an SBAS-on 


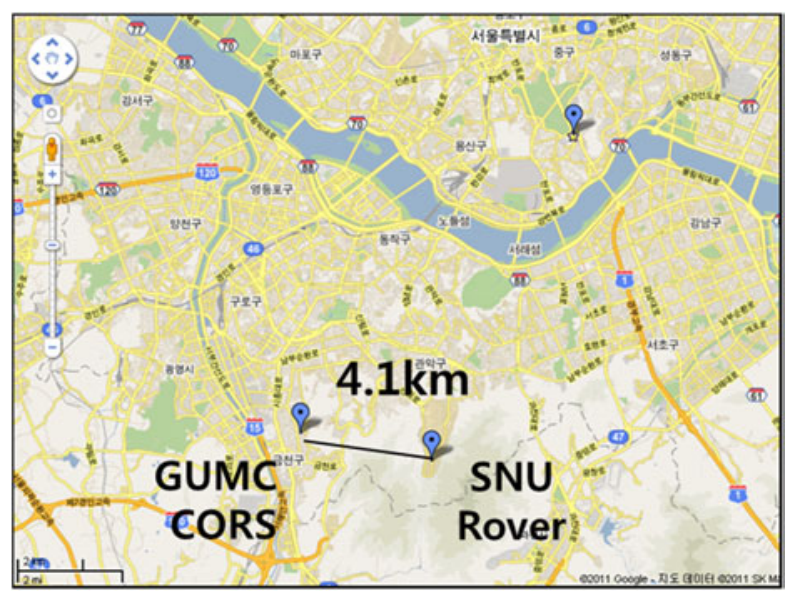

Figure 13. Location of RS and rover for epoch-by-epoch test.

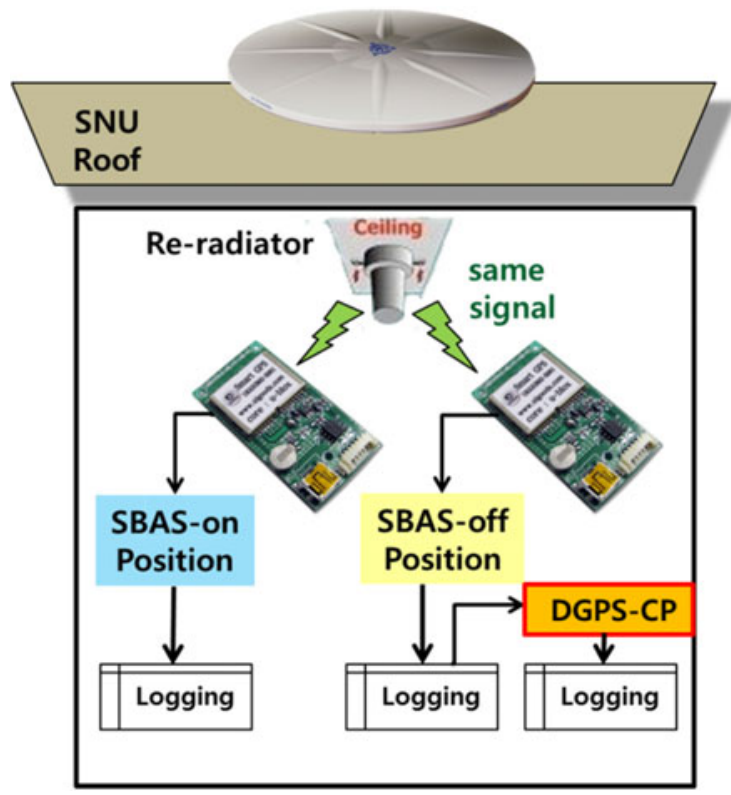

Figure 14. Epoch-by-Epoch DGPS-CP Test Construction.

mode receives the correction from Multi-functional Satellite Augmentation System (MSAS) of Japan.

The rover data was logged in the NMEA format every second with $15^{\circ}$ mask angle from 15:00 to 18:00 (KST: UTC $+9 \mathrm{hr}$ ) in January $3^{\text {rd }}, 2011$, while the range corrections were generated every 15 seconds with $0^{\circ}$ mask at GUMC CORS. The DGPS-CP could be processed inreal-time, but in this test, we used the technique with the SBAS-off logged data to show that it can also be applicable to the alreadycalculated and logged data. As summarized in Figure 15 and Table 5, the DGPS-CP can reduce the horizontal RMS error from $1.25 \mathrm{~m}$ to $0.95 \mathrm{~m}$ and the vertical RMS 
Table 5. Performance statistics of U-Blox DGPS-CP performance (3 hr).

\begin{tabular}{|c|c|c|c|c|}
\hline & \multicolumn{2}{|c|}{ Horizontal Error } & \multicolumn{2}{|c|}{ Vertical Error } \\
\hline & $\operatorname{Mean}(\mathrm{m})$ & $\mathrm{RMS}(\mathrm{m})$ & $\operatorname{Mean}(\mathrm{m})$ & $\mathrm{RMS}(\mathrm{m})$ \\
\hline SBAS off & $0 \cdot 864$ & $1 \cdot 250$ & $-4 \cdot 630$ & $4 \cdot 750$ \\
\hline DGPS-CP & $0 \cdot 259$ & $0 \cdot 946$ & $0 \cdot 287$ & $1 \cdot 090$ \\
\hline SBAS on & $0 \cdot 386$ & $0 \cdot 618$ & $-0 \cdot 314$ & $0 \cdot 593$ \\
\hline
\end{tabular}
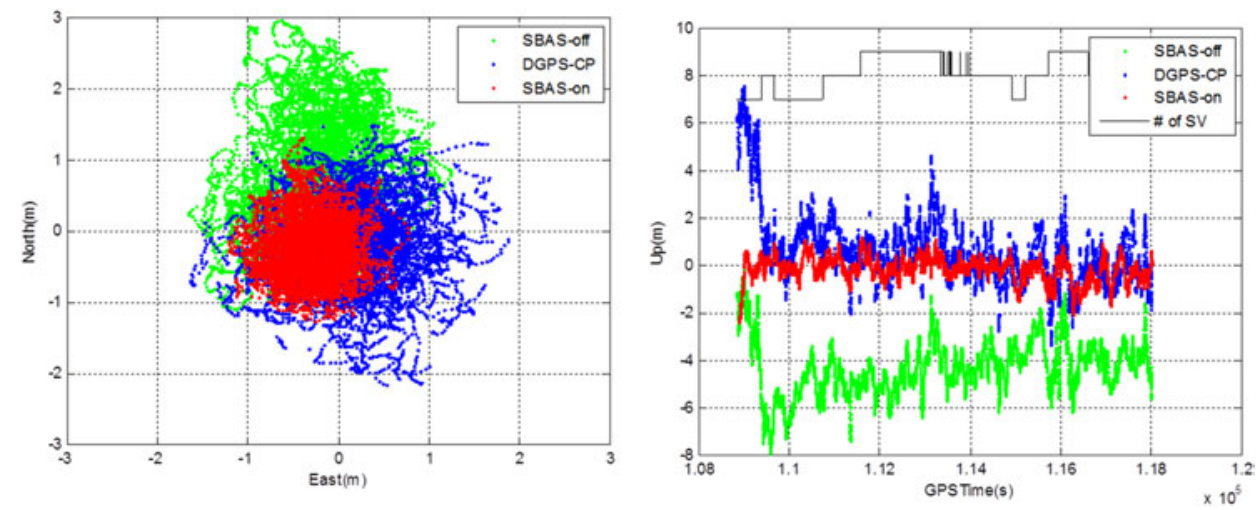

Figure 15. U-Blox DGPS-CP performance (left: Horizontal, right: Vertical).

error from $4.75 \mathrm{~m}$ to $1.1 \mathrm{~m}$. The main contributor to the reduced RMS error is the mitigation of the bias errors, horizontally $0.86 \mathrm{~m}$ and vertically $-4.63 \mathrm{~m}$. An additional note is that the mean error of DGPS-CP is smaller than that of MSAS which is optimal to Japan, not Korea.

7. CONCLUSIONS. Most DGPS correction formats such as Radio Technical Commission for Maritime Services (RTCM), Radio Technical Commission for Aeronautics (RTCA) or Satellite-Based Augmentation System (SBAS) are based on range information, so there is no other way to improve an already-calculated position other than the 'block shift technique' which is far from a practical method. In this paper, we proposed a new Differential Global Positioning System Correction Projection (DGPS-CP) algorithm that corrects Global Positioning System (GPS) errors in a position domain, not in a measurement domain. According to this technique, we can project the range corrections into the position domain data to provide an effective correction of the rover's stand-alone GPS coordinates. If only using GPGGA, GPGSA in the National Marine Electronics Association (NMEA) message and ephemeris navigation information, we can achieve the DGPS-CP system in both post-processing and real-time modes.

To validate the algorithm, we have processed the GPS Continuously Operating Reference Station (CORS) raw data. This showed that DGPS-CP could effectively mitigate the stand-alone position error throughout the entire testing period, while the traditional position-domain DGPS, block-shift was valid in only $50 \%$ of the day. 
Furthermore, the difference between DGPS-CP and the general range-domain DGPS was negligible. We also have analysed the performance of DGPS-CP with U-blox LEA $-5 \mathrm{H}$ receivers. We could mitigate the average errors which were horizontally $0.86 \mathrm{~m}$ and vertically $-4.63 \mathrm{~m}$ to $0.26 \mathrm{~m}$ and $0.28 \mathrm{~m}$, and these results were even better than those of SBAS.

DGPS-CP is able to improve the performance of the low-cost GPS receivers such as in Android phones or car navigation systems which cannot read correction messages. The already-calculated stand-alone position results logged in devices such as digital cameras can be easily corrected by this algorithm. Therefore we expect that DGPS-CP can provide opportunities for DGPS-disabled receiver modules/results to improve position accuracy.

\section{ACKNOWLEDGEMENTS}

This research was supported by a grant from 'Development of GNSS based Transportation Infrastructure Technology (06-A03)' funded by Ministry of Land, Transport and Maritime Affairs of Korean government, contracted through the Institute of Advanced Aerospace Technology at Seoul National University.

\section{REFERENCES}

Kaplan, E. D. (1996). Understanding GPS: Principles and Application. Boston: Artech House Publishers.

Mehaffey, J., Yeazel, J., Penrod, S., and Deiss, A. (2011). NMEA Data. http://www.gpsinformation.org/ dale/nmea.htm. Accessed 30 October 2011.

Misra, P. and Enge, P. (2001). Global Positioning System-Signals, Measurements, and Performance. Ganga-Jamura Press.

NMEA. (2011). NMEA 0183 Standard: http://www.nmea.org/content/nmea_standards/nmea_0183_v_410. asp. Accessed 30 October 2011.

Parkinson, B. (1996). Global Positioning System: Theory and Applications. Progress in Astronautics and Aeronautics.

Payne, A. D. and Carnegie, D. A. (2003). Design and construction of a pair of tricycle based robots to investigate cooperative robotic interaction, Proceedings of Tenth Electronics, New Zealand Conference, Hamilton, New Zealand.

Rizos, C. (1999). How Good is GPS? SNAP-UNSW Website: http://www.gmat.unsw.edu.au/snap/gps/ gps_survey/chap2/244dgps.htm. [Accessed 30 October 2011].

Ublox Inc. (2011). LEA-5 u-blox 5 GPS Modules Data Sheet. Ublox Inc. 